\title{
Scattering Calculations for Asymmetric Raindrops during a Line Convection Event: Comparison with Radar Measurements
}

\author{
Sanja B. Manić, Merhala Thurai, V. N. Bringi, and Branislav M. Notaroš \\ Department of Electrical and Computer Engineering, Colorado State University, Fort Collins, Colorado
}

(Manuscript received 13 November 2017, in final form 20 March 2018)

\begin{abstract}
Two-dimensional video disdrometer (2DVD) data from a line convection rain event are analyzed using the method of moments surface integral equation (MoM-SIE) via drop-by-drop polarimetric scattering calculations at $\mathrm{C}$ band that are compared with radar measurements. Drop geometry of asymmetric drop shapes is reconstructed from 2DVD measurements, and the MoM-SIE model is created by meshing the surface of the drop. The differential reflectivity $Z_{\mathrm{dr}}$ calculations for an example asymmetric drop are validated against an industry standard code solution at $\mathrm{C}$ band, and the azimuthal dependence of results is documented. Using the MoM-SIE analysis on 2DVD drop-by-drop data (also referred to as simply MoM-SIE), the radar variables $\left[Z_{\mathrm{h}}, Z_{\mathrm{dr}}, K_{\mathrm{dp}}, \rho_{\mathrm{hv}}\right]$ are computed as a function of time (with 1-min resolution) and compared to C-band radar measurements. The importance of shape variability of asymmetric drops is demonstrated by comparing with the traditional (or "bulk") method, which uses 1-min averaged drop size distributions and equilibrium oblate shapes. This was especially pronounced for $\rho_{\mathrm{hv}}$, where the MoM-SIE method showed lowered values (dip) during the passage of the line convection consistent with radar measurements, unlike the bulk method. The MoM-SIE calculations of $\left[Z_{\mathrm{h}}, Z_{\mathrm{dr}}, K_{\mathrm{dp}}\right]$ agree very well with the radar measurements, whereas linear depolarization ratio (LDR) calculations from the drop-by-drop method are found to be larger than the values from the bulk method, which is consistent with the dip in simulated and radar-measured $\rho_{\mathrm{hv}}$. Our calculations show the importance of the variance of shapes resulting from asymmetric drops in the calculation of $\rho_{\mathrm{hv}}$ and LDR.
\end{abstract}

\section{Introduction}

Polarimetric weather radars make use of the oblate shapes and high degree of orientation of raindrops in order to better estimate rainfall rates from the retrieved raindrop size distributions (Seliga and Bringi 1976, 1978). As a first step, they utilize the differential reflectivity $\left(Z_{\mathrm{dr}}\right)$ along with the conventionally measured copolar horizontal reflectivity $\left(Z_{\mathrm{h}}\right)$ to reduce uncertainties in estimating the drop size distribution within the radar pulse volume (Bringi and Chandrasekar 2001).

The equilibrium shapes of raindrops are size dependent (Beard and Chuang 1987), and they are generally approximated by oblate spheroids whose axis ratios (minor to major) decrease monotonically with increasing size. Drops with diameters smaller than $0.8 \mathrm{~mm}$ can be considered almost spherical. For larger drops $(>2.5 \mathrm{~mm})$ the concept of dynamic equilibrium shape was introduced by Szakáll et al. (2009) to describe the time-averaged axis ratios resulting from drop

Corresponding author: Merhala Thurai,merhala@colostate.edu oscillations observed in a wind tunnel, which was also confirmed using the concept of "most probable" shapes from a 2D video disdrometer (2DVD; Schönhuber et al. 2008) by Thurai et al. (2009a). It is now well known that axisymmetric drop oscillations dominate the background state with smaller amplitude mixed oscillation modes that give rise to asymmetric shapes (Beard et al. 2010). However, there is no theoretical framework for modeling such asymmetric shapes in natural rainfall. Hence, the common approach is to neglect the variance of drop shapes and to relate the mean axis ratio with drop equivolume diameter $\left(D_{\text {eq }}\right)$ (Beard and Chuang 1987; Brandes et al. 2004; Thurai et al. 2007).

Asymmetric drops were inferred from 2DVD measurements in a highly organized line convection rain event described in Thurai et al. (2013). During this event a significant fraction of drops (around 30\%) within the line convection were asymmetric. Eight individual asymmetric drops were

Publisher's Note: This article was revised on 14 June 2018 to include higher resolution versions of Figs. 4, 6, and 8 to replace the lower quality versions that were originally published. 
chosen from the 2DVD measurements to reconstruct their 3D shapes (Thurai et al. 2017) and to determine their individual scattering amplitudes at $\mathrm{C}$ band using the method of moments in the surface integral equation (MoM-SIE) formulation (Chobanyan et al. 2015). Scattering calculations for the individual asymmetric drops showed that the single- $Z_{\mathrm{dr}}$ values differed from those calculated assuming rotationally symmetric shapes. Differences were also seen in the case of (single particle) specific differential phase $\left(K_{\mathrm{dp}}\right.$ factor) as well as linear depolarization ratio (LDR).

Accurate simulations of radar observables require accounting for variance in drop shapes, which requires the computation of drop-by-drop scattering amplitudes and the integration of the elements of the covariance matrix over a given measurement interval (Bringi and Chandrasekar 2001). The aforementioned line convection event is analyzed in this manner herein using 3D-reconstruction of drop shapes from 2DVD data. The radar reflectivity, the differential reflectivity, the copolar correlation coefficient, and the specific differential phase are computed with 1-min time resolution and compared to radar measurements extracted over the 2DVD site from the University of Alabama in Huntsville Advanced Radar for Meteorological and Operational Research (ARMOR) C-band radar (see Petersen et al. 2007; Crowe et al. 2012). The radar is $15 \mathrm{~km}$ away from the ground instrument site, and the height of the resolution volume is around $340 \mathrm{~m}$ at the lowest elevation angle of $1.3^{\circ}$. For completeness the linear depolarization ratio is also computed even though the radar is not configured for measuring LDR. The drop-by-drop scattering simulations are compared with the bulk method, which refers to the use of the T-matrix scattering code (Barber and Yeh 1975) with input being the 1-min averaged drop size distributions from 2DVD and the oblate axis ratios from Thurai et al. (2007) with Gaussian canting angle distribution [mean $=0^{\circ}, \sigma=5^{\circ}$ ].

This paper is organized as follows. In section 2 we revisit the methodology used to obtain the scattering results. Section 3 is reserved for the details of raindrop model construction, used as input to the scattering code. In section 4 , for validation purposes, we consider an example of an asymmetric drop that is reconstructed from 2DVD measurements (Thurai et al. 2017), and the results obtained by our electromagnetic solver (MoM-SIE) are compared to those using an industry standard software. We then consider, in section 5, the line convection event and compare radar measurements with scattering simulations using the drop-by-drop method as well as the bulk method. The paper concludes with a short discussion and conclusions section.

\section{Methodology: Numerical solution}

Raindrop scattering calculations assuming oblate (or rotationally symmetric) shapes typically use the T-matrix method (Waterman 1965; Barber and Yeh 1975; Mishchenko et al. 1996), which is widely used by the radar meteorology community [see, also, Chobanyan et al. (2015) and references therein for a review of different scattering methodologies, including discrete dipole approximation, and surface and volume integral formulations used for precipitation particles].

Scattering calculations are performed herein using a higher-order method of moments solution to the electric and magnetic field surface integral equations (MoM-SIE) based on boundary conditions between an air and water dielectric at the raindrop surface $S_{a}$, that is, the continuity of tangential components of total (incident plus scattered) electric/magnetic fields (Notaroš 2008; Djordjević and Notaroš 2004)

In our current work using the MoM-SIE methodology, a geometrical model is obtained by discretization of the raindrop surface using Lagrange-type curved parametric quadrilateral elements of arbitrary orders (Djordjević and Notaroš 2004; Chobanyan et al. 2015). The method directly solves for an approximation of fictitious surface electric and magnetic current densities, $\mathbf{J}_{s}$ and $\mathbf{M}_{s}$, respectively, over the raindrop boundary using hierarchical divergenceconforming polynomial basis functions, defined over quadrilateral elements (Djordjević and Notaroš 2004; Chobanyan et al. 2015).

For a given incident wave, the scattered electric field is represented as the following function of current densities:

$$
\begin{aligned}
\mathbf{E}^{\mathrm{scat}}\left(\mathbf{J}_{s}, \mathbf{M}_{s}, \varepsilon\right)= & -j \omega \mu_{0} \int_{S_{a}}\left(\mathbf{J}_{s} g+k^{-2} \nabla_{s} \cdot \mathbf{J}_{s} \nabla g\right) d S_{a} \\
& +\int_{S_{a}} \mathbf{M}_{s} \times \nabla g d S_{a}
\end{aligned}
$$

where $g\left(g=e^{-\mathrm{j} k R} / 4 \pi R\right)$ and $k \quad\left(k=\omega \sqrt{\varepsilon \mu_{0}}\right)$, respectively, are Green's function and the wavenumber for the unbounded medium of parameters $\varepsilon=\varepsilon_{r} \varepsilon_{0}$ and $\mu_{0}$, with $R$ being the distance of the field point from the source point, $\omega=2 \pi f$ is the angular (radian) frequency, and $\varepsilon_{r}$ is the dielectric constant of the raindrop (water). The magnetic field is expressed in a similar fashion.

When the distance $R$ in (1) is zero or relatively small, the singular or near-singular terms are extracted and evaluated analytically, and the remaining nonsingular integrals are calculated numerically using Gauss-Legendre integration formulas. The final matrix equation is obtained after the Galerkin testing procedure has been applied to boundary condition equations, which assumes another surface integration of the SIEs with testing (weighting) functions being equal to the basis functions. 
For verification purposes, another method that utilizes $3 \mathrm{D}$ geometrical discretization is considered and the results are presented in terms of single-particle dual-polarization scattering for three different frequency bands.

\section{Raindrop modeling}

Drop shapes recorded by the 2DVD are used for $3 \mathrm{D}$ reconstruction (for $D_{\text {eq }}>2 \mathrm{~mm}$ ) using the algorithm in Schönhuber et al. (2016). Drops with $D_{\text {eq }}<2 \mathrm{~mm}$ are assumed to have oblate spheroidal shapes with an axis ratio as a function of $D_{\text {eq }}$ given in Thurai et al. (2007). The 3D-reconstruction procedure gives rise to more significant errors for small drops (resulting from a resolution of $170 \mu \mathrm{m}$ ) and hence the 2-mm threshold was applied. Note also that the larger drops will have more of an effect on $Z_{\mathrm{dr}}$ than the small drops.

Details of the 3D shape reconstruction of each recorded drop from its images from the two orthogonal cameras have been published previously (Schönhuber et al. 2016; Schwinzerl et al. 2016); hence, only a brief summary is given here. The 2DVD measures drop contours in two perpendicular planes that can be skewed because of the horizontal component of the drop velocity (typical in line scan camera systems). For drops that possess an axis of symmetry, the contours can be deskewed as described in Schönhuber et al. (2000) and Huang et al. (2008); in addition, the horizontal velocity can be estimated. In the appendix of Thurai et al. (2017), the horizontal drop velocities derived from the deskewing procedure were shown to be in excellent agreement with the independent wind sensor measurements, in both magnitude and direction. For deskewing asymmetric drops, the horizontal velocity must be estimated. This is achieved from the drop horizontal velocities estimated from the deskewed symmetric drops closest in time and size to the asymmetric drop (see section IV of Schönhuber et al. 2016). One limitation of this method for asymmetric drops relates to the uncertainty in the exact drop horizontal velocity required as input to the deskewing procedure. However, apart from errors caused by rapid fluctuations in wind velocities, we expect the reconstructed shapes to be reasonably representative of their true "instantaneous" shapes.

The deskewed contours in the two orthogonal planes are sampled at equidistant values along the vertical axis, and four points are obtained at each height (note that for rotationally symmetric drops, the thin "slices" along the vertical axis are elliptical and the 3D shape is based on stacked ellipses). For asymmetric drops, four different ellipse quarters are constructed for each slice having in mind the center point. The points describing the geometry of each slice are obtained by sampling the constructed elliptical quarters in uniform intervals of the

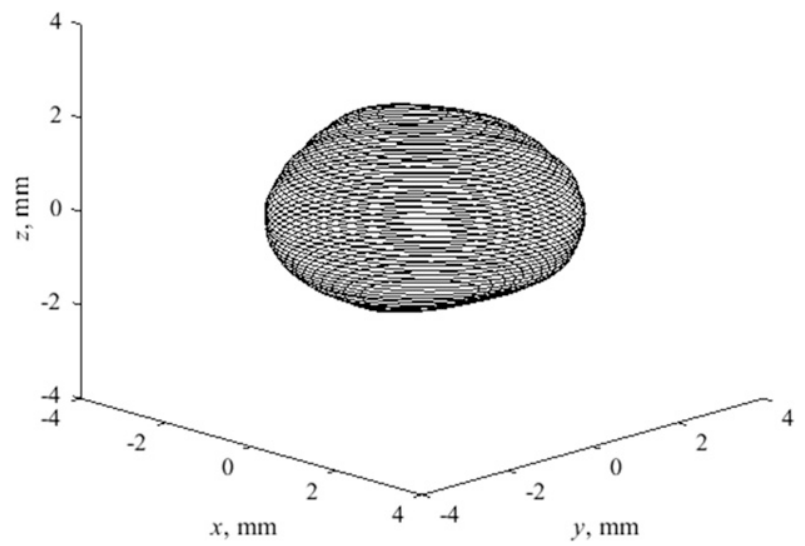

FIG. 1. Reconstructed drop from 2DVD measurements in natural rain (equivolume drop diameter $=4.81 \mathrm{~mm}$ ).

azimuth angle. The procedure is repeated for each slice in order to create the 3D-reconstructed drop, an example of which is shown in Fig. 1. In our models, the shapes of the slices are limited to convex shapes; that is, the center point needs to be inside or part of the circumference.

The model of the drop is created by defining firstorder (bilinear) quadrilateral elements, each between four points of the geometry (Thurai et al. 2017). To define one element, two points are chosen with the same coordinate value on the $z$ axis and sequential values on the azimuthal coordinate. Two other points are chosen to have the same azimuth angles but different, consecutive $z$ axis values compared to the two already chosen points. After creating all the elements by connecting pairs of points from groups with consecutive values on the $z$ axis, the elements at the top and the bottom of the drop are defined using all four points from the group having the same $z$ axis value, the highest and the lowest, respectively, so the entire surface of the drop is discretized. The order of the basis functions (Djordjević and Notaroš 2004) used for the unknown expansion over the elements was chosen to comply with Klopf et al. (2012).

\section{Validation of the MoM-SIE method}

The single-particle differential reflectivity $Z_{\mathrm{dr}}$ (expressed as a ratio) is given by

$$
Z_{\mathrm{dr}}=\frac{\left|S_{\mathrm{hh}}\right|^{2}}{\left|S_{\mathrm{vv}}\right|^{2}},
$$

where $S_{\mathrm{hh}}$ and $S_{\mathrm{vv}}$ are the frequency-dependent backscatter amplitudes for horizontal (h) and vertical (v) polarizations. Figure 2 shows the calculated $Z_{\mathrm{dr}}$ for the 


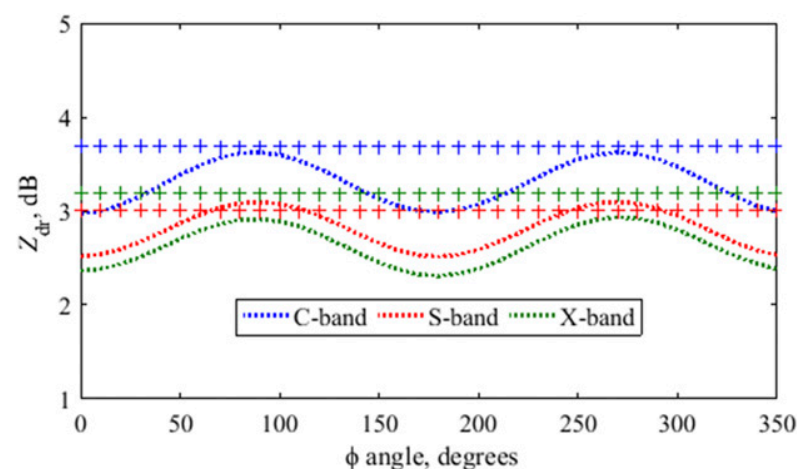

FIG. 2. Variation of $Z_{\mathrm{dr}}(\mathrm{dB})$ with $\phi$, in horizontal plane, for C, S, and $\mathrm{X}$ bands for the reconstructed drop in Fig. 1 (dotted lines). The corresponding $Z_{\mathrm{dr}}$ values for the most probable shapes (plus signs).

reconstructed drop in Fig. 1 as a function of the ("look") azimuthal angle $\phi$, for the S, C, and X bands. In all three cases, the $Z_{\mathrm{dr}}$ variation with $\phi$ is significant, whereas for a rotationally symmetric drop the $Z_{\mathrm{dr}}$ is $\phi$ independent, with values of 3.0,3.7, and $3.2 \mathrm{~dB}$ for the $\mathrm{S}, \mathrm{C}$, and $\mathrm{X}$ bands, respectively, marked as "+" points. Figure 2 also shows that the C-band variation lies well above the S- and X-band variations, which can be attributed to this particular drop size $\left(D_{\text {eq }}=4.81 \mathrm{~mm}\right)$ lying in the C-band resonance scattering region (e.g., Carey and Petersen 2015). The $\phi$ angle variation at C band is also slightly higher than those at $\mathrm{S}$ and $\mathrm{X}$ bands.

Although Fig. 2 shows a somewhat periodic variation with the $\phi$ angle for all three frequency bands, the real and imaginary parts of $S_{\mathrm{hh}}$ and $S_{\mathrm{vv}}$ do not necessarily show the same trend. As an example, Fig. 3 shows these variations for C band. The imaginary parts of $S_{\mathrm{hh}}$ and $S_{\mathrm{vv}}$ show nonperiodic variations, but their amplitudes are considerably lower than the corresponding real parts. It turns out that the $Z_{\mathrm{dr}}$ variation is much more governed by the variation in $\operatorname{Re}\left(S_{\mathrm{hh}}\right)$ and $\operatorname{Re}\left(S_{\mathrm{vv}}\right)$ than by $\operatorname{Im}\left(S_{\mathrm{hh}}\right)$ and $\operatorname{Im}\left(S_{\mathrm{vv}}\right)$.

By way of verification of the MoM-SIE-based scattering amplitude results, another method that uses 3D discretization, namely, the ANSYS High Frequency Structural Simulator (HFSS) $\operatorname{code}^{1}$ [ANSYS 2017; industry standard utilizing the volumetric finite element method (FEM), so numerically very different from the MoM-SIE approach], is employed. Results by the FEM (HFSS) with the computational region truncated by means of a perfectly matched layer (PML) are also included in Figs. 3a and 3b. As can be seen, the resulting scattering amplitudes are very close to the MoM-SIEbased results. MoM-SIE methods are computationally

\footnotetext{
${ }^{1}$ See http://www.ansys.com/products/electronics/ansys-hfss.
}
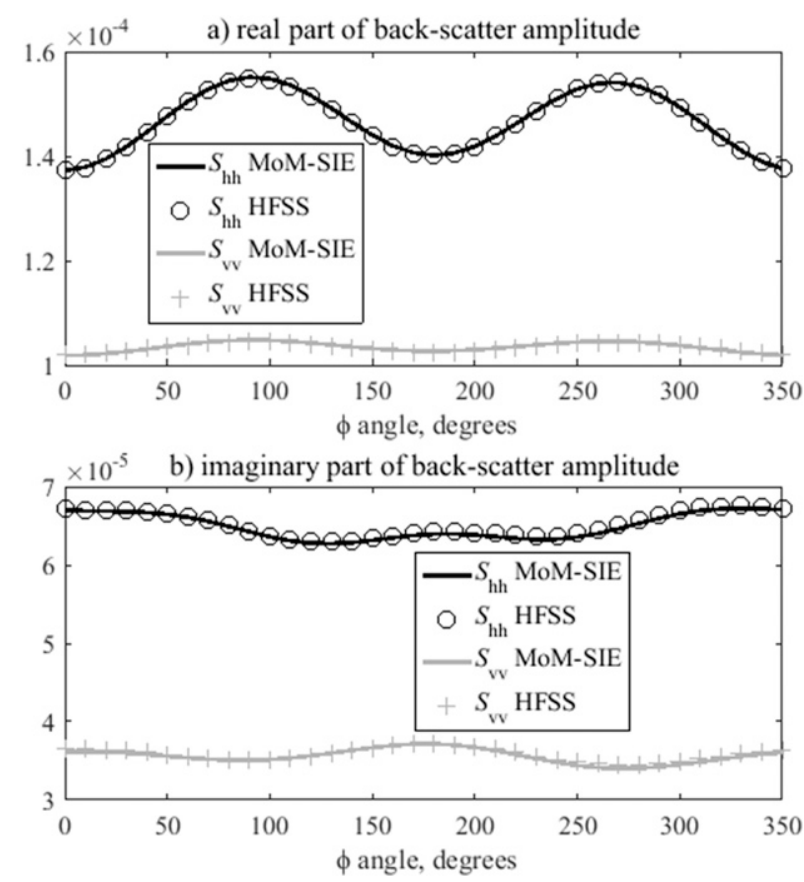

FIG. 3. (a) Real part and (b) imaginary part of C-band backscatter amplitudes as a function of $\phi$, in horizontal plane, for $\mathrm{h}$ and $\mathrm{v}$ polarizations, for the reconstructed drop given in Fig. 1. Computations using MoM-SIE and HFSS-FEM methods are displayed.

efficient for electromagnetic problems with a small volume-to-surface ratio and when Green's function can be calculated. ${ }^{2}$ FEM-based codes are widely used in computational electromagnetics (in industry), but they require discretization of the whole three-dimensional domain as well as region truncation with boundary condition in order to compute far-field scattering results that are easily computed by the SIE method.

\section{Calculation of radar variables and comparisons with radar measurements}

We now consider the rain event that occurred in Huntsville, Alabama, on 25 December 2009. This was a widespread event with an embedded line convection that traversed the disdrometer site (Thurai et al. 2013). The 2DVD measurements for this event showed that a significant fraction of the drops within the line convection (around $30 \%$ ) did not possess any rotational symmetry axis (i.e., asymmetric).

Altogether, 2DVD measurements over a period of 100 min were analyzed during which there were 114317 drops recorded by the instrument, out of which 10233 drops had $D_{\text {eq }} \geq 2 \mathrm{~mm}$. For all the drops with

\footnotetext{
${ }^{2}$ See https://en.wikipedia.org/wiki/Computational_electromagnetics.
} 

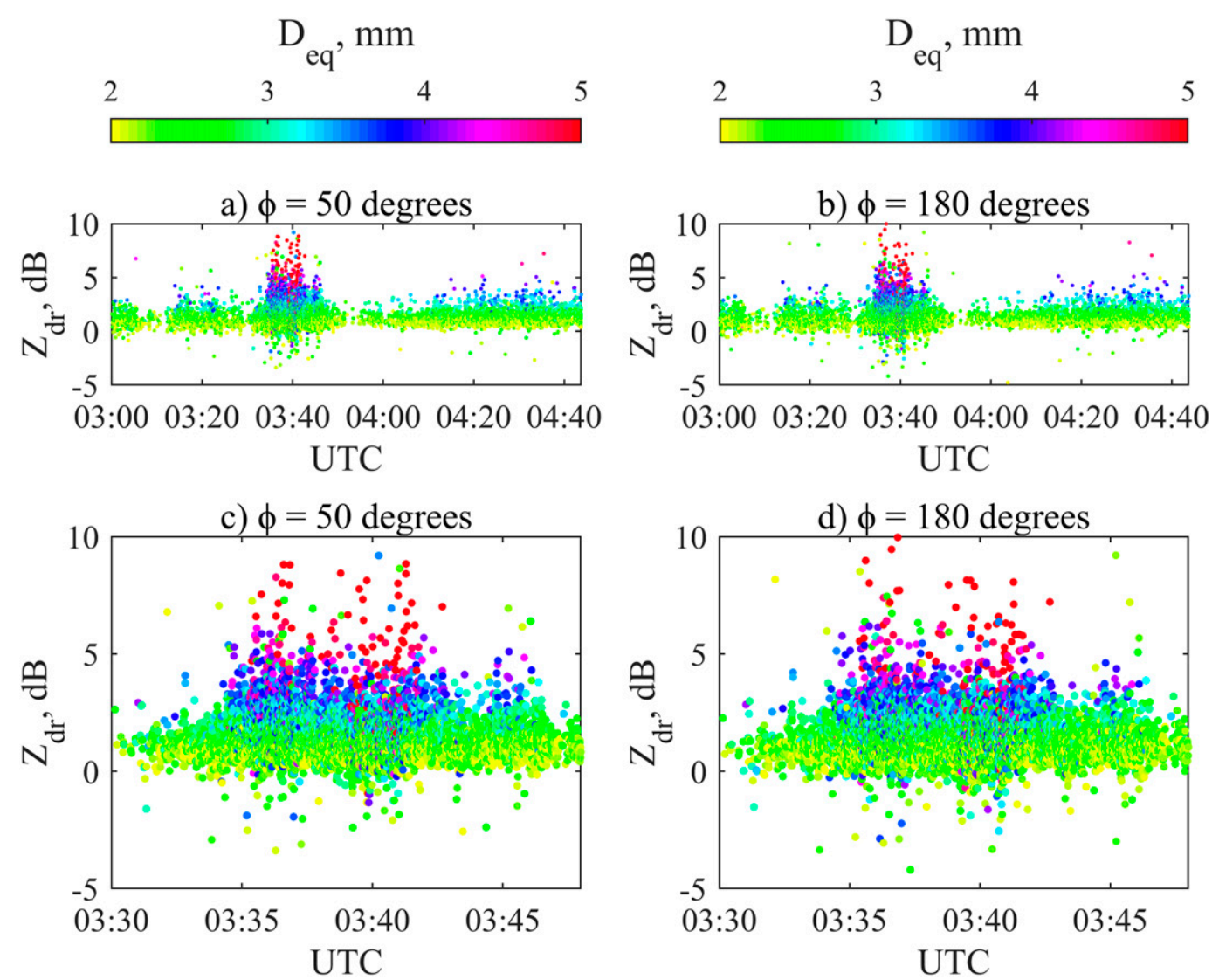

FIG. 4. Single-particle $Z_{\mathrm{dr}}$ for all drops with $D_{\mathrm{eq}}>2 \mathrm{~mm}$ (a) from 0300 to $0440 \mathrm{UTC}$ for $\phi=50^{\circ}$. (b) As in (a), but for $\phi=180^{\circ}$. (c),(d) Zoomed-in versions of (a) and (b), respectively, during the passage of the line convection over the disdrometer site. In all cases the points are color coded according to the drop size.

$D_{\text {eq }} \geq 2 \mathrm{~mm}$, the 3D shapes were constructed in the same way as outlined in Thurai et al. (2017), and their individual scattering amplitudes were calculated using the MoM-SIE method. The individual particle $Z_{\mathrm{dr}}$ is plotted as a time series in Fig. 4 for two values of the incident angle. The top two panels show the $Z_{\mathrm{dr}}$ for all drops with $D_{\text {eq }} \geq 2 \mathrm{~mm}$ for the entire 100-min period, while the two lower panels show the same but for the zoomed-in period. In all cases the drop sizes are color coded. The variability in $Z_{\mathrm{dr}}$ for a given drop size is particularly evident for the large drops $(>4 \mathrm{~mm})$ and, further, the dependence on the azimuthal angle is also evident. For all drops with $D_{\text {eq }}<2 \mathrm{~mm}$, the oblate shapes were assumed whose axis ratios were determined using the diameter-dependent relationship given in (2) of Thurai et al. (2007). For these drops the individual scattering amplitudes were also computed using the MoM-SIE method. The variability of the single-particle $Z_{\mathrm{dr}}$ during the line convection passage is evident from Fig. 4c though some of the variability is due to sampling errors for the larger sizes, which are much lower in concentration. With this consideration the variability in single-particle $Z_{\mathrm{dr}}$ for a given $D_{\text {eq }}$ reflects the variance in shapes that is partly due to the asymmetric drops. The coefficient of variation of $Z_{\mathrm{dr}}$ (expressed as a ratio) for sizes $>3 \mathrm{~mm}$ is around 0.5 . The coefficient of variation of the "effective" axis ratio is then $\approx 0.2$ using the approximate formula from Jameson (1983). The deduced axis ratio variability is around twice that found by Thurai et al. (2009a) as a result of asymmetric drops.

From the backscatter amplitudes of each individual drop over a finite period $(1 \mathrm{~min})$ and the drop-by-drop integration of the relevant covariance matrix elements (Bringi and Chandrasekar 2001), the radar reflectivity for horizontal polarization $\left(Z_{\mathrm{h}}\right)$, differential reflectivity $\left(Z_{\mathrm{dr}}\right)$, and copolar correlation coefficient $\left(\rho_{\mathrm{hv}}\right)$ were computed, for comparisons with the C-band ARMOR radar measurements [see (3)-(6), later in the text]. This method will also be referred to as the MoM-SIE. Note that for $K_{\mathrm{dp}}$ calculation, the forward scatter amplitudes are used. The finite period chosen here is $1 \mathrm{~min}$, since for a smaller averaging period the sampling errors will be large (Schuur et al. 2001) and for a larger averaging period drop sorting errors will also be large (Lee and Zawadzki 2005). Note from Figs. $4 \mathrm{c}$ and $4 \mathrm{~d}$ that 

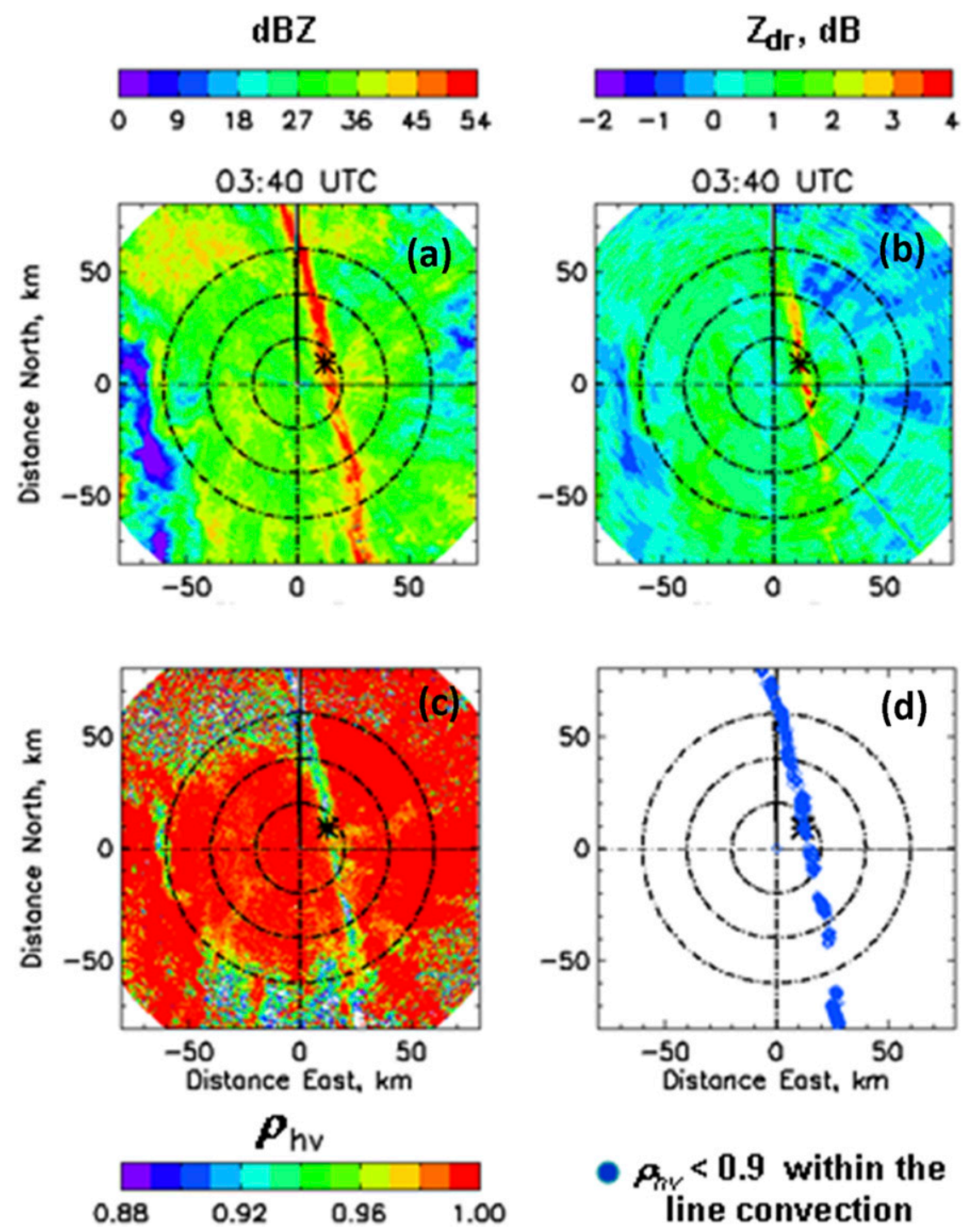

FIG. 5. PPI scans of (a) attenuation-corrected $Z_{\mathrm{h}}$, (b) attenuation-corrected $Z_{\mathrm{dr}}$, and (c) $\rho_{\mathrm{hv}}$, taken at (top to bottom) 0340 UTC when the line convection was directly above the 2DVD site (marked with an asterisk along azimuth $52^{\circ}$ and range $15 \mathrm{~km}$ ). (d) The areas within the line convection where $\rho_{\mathrm{hv}}$ values were lower than 0.9 .

the line convection passage over the disdrometer site took around $15 \mathrm{~min}$, from 0333 to 0348 UTC.

Figure 5 shows the plan position indicator (PPI) scan taken with the ARMOR radar (Petersen et al. 2007) at an elevation angle of $1.3^{\circ}$. The time of the scan was 0340 UTC. The "star" mark represents the location of the 2DVD, and at this time the line convection was directly positioned over the disdrometer site. Figures $5 \mathrm{a}$ and $5 \mathrm{~b}$ show the copolar reflectivity and the differential reflectivity after correcting for attenuation and differential attenuation, respectively. The correction procedures use the specific differential propagation phase-based algorithms, using the same procedure described in Bringi et al. (2006).
The reflectivity values were high at the site $(>50 \mathrm{dBZ})$ and the differential reflectivity values were also high $(>4 \mathrm{~dB})$, indicating large drops in the strong precipitation shaft. Other PPI scans taken before and after 0340 UTC can be seen in Fig. 7 in Thurai et al. (2013). Figure 5c shows the corresponding copolar correlation coefficient, $\rho_{\text {hv }}$, and Fig. $5 \mathrm{~d}$ marks the areas within the line convection where $\rho_{\text {hv }}$ was less than 0.9. Values of attenuation-corrected $Z_{\mathrm{h}}$ and $Z_{\mathrm{dr}}$, as well as $\rho_{\mathrm{hv}}$, were extracted near and around the radar pixels surrounding the 2DVD site $(14.5-\mathrm{km}$ radar range, $52.7^{\circ}$ azimuth) from all the PPI sweeps that were taken from 0300 to 0440 UTC. For a given elevation angle, each sweep was repeated at 5-min intervals. 

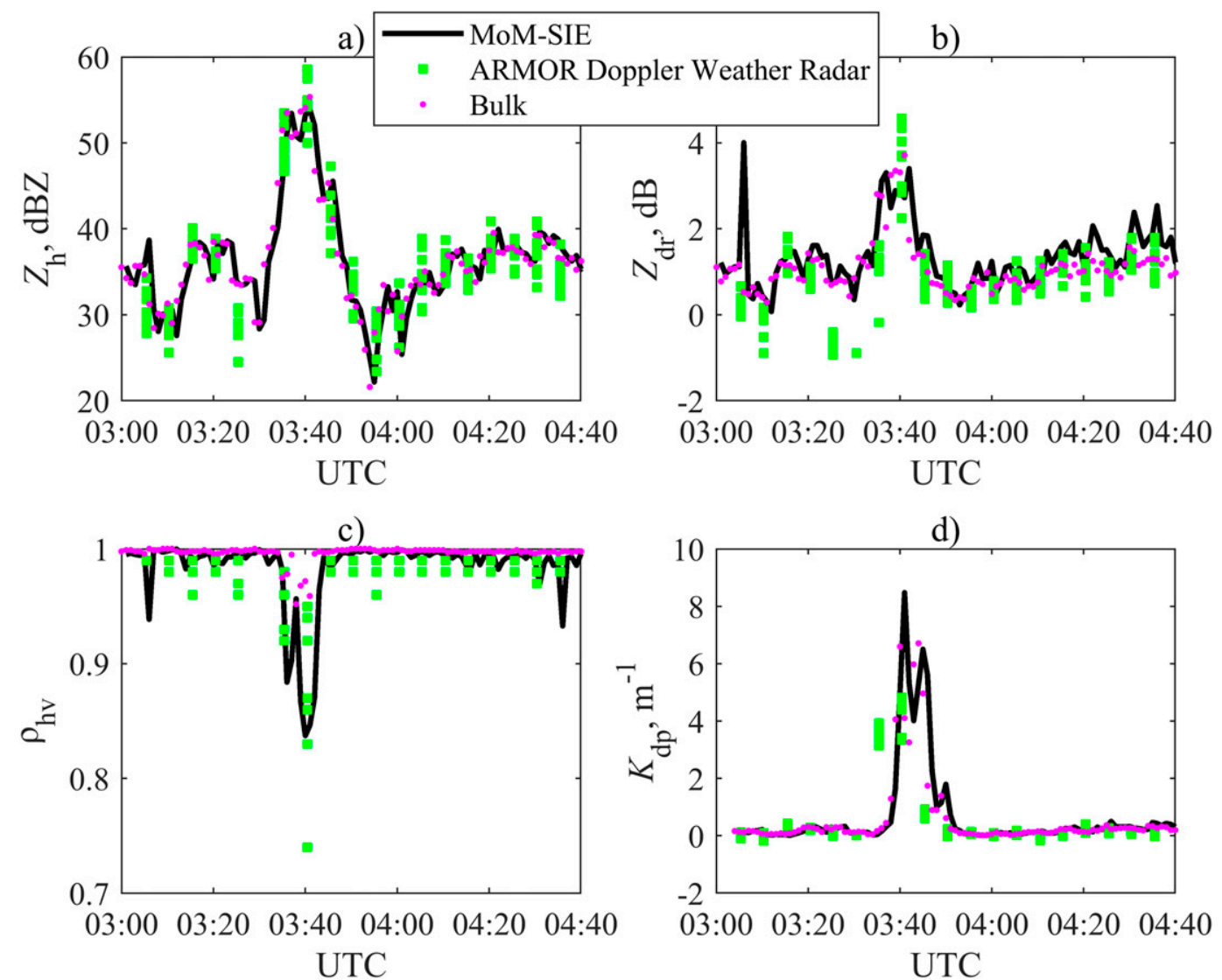

FIG. 6. Polarimetric radar variables comparison between MoM-SIE, bulk method, and radar measurements: (a) Reflectivity $Z_{\mathrm{h}}$, (b) differential reflectivity $Z_{\mathrm{dr}}$, (c) copolar correlation coefficient $\rho_{\mathrm{hv}}$, and (d) specific differential propagation phase $K_{\mathrm{dp}}$.

The extracted $Z_{\mathrm{h}}$ and $Z_{\mathrm{dr}}$ are shown in Figs. 6a and 6b, respectively, for the 100 -min period. For a given PPI sweep time, several points are shown that correspond to the "2DVD pixel" and to the "immediately adjacent" pixels in both azimuth and range, covering an area of approximately $750 \mathrm{~m} \times 750 \mathrm{~m}$ over the 2DVD site. Altogether, 20 PPI sweeps were used over the entire 100-min period. Reflectivity and differential reflectivity values reach their highest values at 0340 UTC. Later on, at around 0430 UTC, reflectivity values again rise but only up to $40 \mathrm{dBZ}$. Differential reflectivity remains relatively low, indicating that the maximum drop sizes were significantly lower at 0430 than at 0340 UTC. The measured drop size distributions (DSDs) can be seen in Fig. $2 b$ in Thurai et al. (2013). At 0340, the spectra showed the highest massweighted mean diameter and the highest standard deviation of the mass spectrum (not shown).

Overplotted in black in Figs. $6 \mathrm{a}$ and $6 \mathrm{~b}$ are the $Z_{\mathrm{h}}$ and $Z_{\mathrm{dr}}$ calculations, respectively, based on the individual scattering amplitudes of drops (i.e., drop-by-drop integration using MoM-SIE or simply MoM-SIE) over each 1-min period. The radar measurements of $Z_{\mathrm{h}}$ and $Z_{\mathrm{dr}}$ show good temporal correlation and agreement with the MoM-SIE as well as bulk calculations with the radar peak values being somewhat larger $(60 \mathrm{dBZ}$ and $4 \mathrm{~dB})$ than the simulations perhaps because of disdrometer sampling limitations for large drops or the applied smoothing. While the agreement between the MoM-SIE method and the bulk method for $Z_{\mathrm{h}}$ is expected, the agreement of $Z_{\mathrm{dr}}$ is somewhat unexpected given the large variance in individual drop $Z_{\mathrm{dr}}$ values in the line convection region (see Fig. 4c), especially for the large drops. The bulk method $Z_{\mathrm{dr}}$ is essentially related to the reflectivity-weighted mean axis ratio, which would equal the drop-by-drop integrated $Z_{\mathrm{dr}}$ if the axis ratio distribution is narrow (Jameson 1983; Bringi and Chandrasekar 2001). As discussed earlier, the coefficient of variation of the effective axis ratio in the line convection is estimated to be around a factor of 2 larger than the value from Thurai et al. (2009a), which is based on data from an artificial rain experiment where asymmetric drops were not detected. In spite of this 
increase, the axis ratio distribution in the line convection case is judged to be narrow enough that the drop-bydrop MoM-SIE-computed $Z_{\mathrm{dr}}$ is in good agreement with the bulk method.

Note the radar reflectivity for an individual (ith) drop, in a volume of $1 \mathrm{~m}^{3}$, is given by

$$
Z_{i}=10^{18} \frac{\lambda^{4}}{\pi^{5}\left|K_{w}\right|^{2}} \eta_{i},
$$

where $\lambda$ is the wavelength in air, $\eta_{\mathrm{h} / \mathrm{v}}=4 \pi\left|S_{\mathrm{hh} / \mathrm{vv}}\right|^{2}$ is backscatter cross section per unit volume for $\mathrm{h} / \mathrm{v}$ polarization, and $K_{w}=\left(\varepsilon_{r}-1\right)\left(\varepsilon_{r}+2\right)^{-1}=0.9631-j 0.0111$ is the dielectric factor of water at $\mathrm{C}$ band with dielectric constant $\varepsilon_{r}=72.5-j 22.43$. Over a 1 -min period, the resulting reflectivity $Z$ is derived by summing the individual drop reflectivities and is calculated using

$$
Z=\frac{1}{A \Delta t} \sum_{i} v_{i}^{-1} Z_{i}
$$

where $A$ is the measurement area of the 2DVD, $\Delta t$ is the averaging period, and $v_{i}$ is the vertical velocity of the $i$ th drop. Equations (3) and (4) are used to evaluate the overall radar reflectivity based on the individual scattering amplitudes for each of the reconstructed raindrops as well as their individual measured fall velocities. The computed $Z$ values for $\mathrm{h}$ and $\mathrm{v}$ polarizations are converted to the conventional $\mathrm{dB} Z$ units, and $Z_{\mathrm{dr}}(\mathrm{dB})$ is obtained from the difference between the two.

Figure $6 \mathrm{c}$ shows the calculated $\rho_{\mathrm{hv}}$ values using

$$
\rho_{\mathrm{hv}}=\frac{\left|\sum_{i} v_{i}^{-1} S_{\mathrm{hh}}^{*} S_{\mathrm{vv}}\right|}{\sqrt{\sum_{i} v_{i}^{-1}\left|S_{\mathrm{hh}}\right|^{2} \sum_{i} v_{i}^{-1}\left|S_{\mathrm{vv}}\right|^{2}}}
$$

where $v_{i}$ is the vertical velocity of the $i$ th drop, $S$ represents single-drop backscattering amplitude, and the summation is done over all the drops recorded by the 2DVD during the considered time interval. From 0335 to 0340 UTC, a sharp decrease or dip in $\rho_{\text {hv }}$ is seen, reaching as low as 0.8 . Such low values are consistent with the radar measurement of $\rho_{\mathrm{hv}}$ as low as 0.85 in the PPI plot in Fig. 5d. For comparison, C-band scattering calculations using the 1-min averaged DSDs and bulk assumptions are included in magenta in Fig. 6c. The lowest value using the bulk assumptions is only 0.96 . Clearly, the drop-by-drop MoM-SIE-based calculations give rise to much more accurate $\rho_{\text {hv }}$ predictions than the bulk method. This is due to the inability of the bulk method to capture the variability of drop shapes during the line convection passage. Note, however, that at other times - that is, prior to 0335 and after 0345 UTC - both methods predict $\rho_{\mathrm{hv}}$ values that are close to 1 . These values are consistent with radar measurements over the 2DVD site at these other times. The measurement accuracy of $\rho_{\mathrm{hv}}$ is around $1 \%$, which is substantially less than the simulated change from 0.96 to $0.8-0.85$, so the dip should be detectable if the SNR $>20 \mathrm{~dB}$ or so (Bringi and Chandrasekar 2001).

Figure $6 \mathrm{~d}$ compares the specific differential propagation phase $\left(K_{\mathrm{dp}}\right)$ derived from the ARMOR range profiles of the differential phase $\left(\Phi_{\mathrm{dp}}\right)$ with the corresponding scattering calculations. For the radar-based $K_{\mathrm{dp}}$ values, the finite impulse response (FIR) range filtering technique is used, as described in Hubbert and Bringi (1995), having the advantage of quantifying and removing any backscatter differential phase contribution, which at $\mathrm{C}$ band can become significant when large drops or small melting hail are present in the radar pulse volume. However, close examination of the phase data showed the backscatter differential phase $\delta<3^{\circ}-5^{\circ}$ along the line convection, which discounts the presence of small melting hail for which $\delta$ could reach $20^{\circ}$ (Meischner et al. 1991). For the scattering calculations, as in other panels of Fig. 6, the bulk calculations (assuming rotational symmetry) are shown in magenta and the MoM-SIE calculations are shown as a black line. Term $K_{\mathrm{dp}}$ is calculated from

$$
K_{\mathrm{dp}}=10^{3} \lambda \frac{180}{\pi} \frac{1}{A \Delta t} \sum_{i} v_{i}^{-1} \operatorname{Re}\left[S_{\mathrm{hh}}-S_{\mathrm{vv}}\right]
$$

where $S$ represents forward scattering amplitudes. The summation is done over all drops recorded in the considered time interval. The bulk calculations are in good agreement with the MoM-SIE calculations, which indicates that $K_{\mathrm{dp}}$ is not dependent on the variance of shapes; rather, it is related to the mass-weighted mean axis ratio (Jameson 1985; Bringi and Chandrasekar 2001). The radar estimate of $K_{\mathrm{dp}}$ is smaller than the calculations because of the range filtering and smoothing methodology used across the compact line convection region.

In Thurai et al. (2017), the cross-polar backscatter from asymmetric drops in terms of single-particle LDR was also considered. Here we extend to drop-by-drop MoM-SIE LDR calculations as the ratio of the crosspolar reflectivity to the copolar reflectivity and compare that with the bulk method as shown in Fig. 7. It is immediately clear that during the line convection passage, the MoM-SIE method shows a much larger LDR than the bulk method (peak of $-17 \mathrm{~dB}$ vs $-26 \mathrm{~dB}$ ). Even outside the line convection, the MoM-SIE LDR is larger by $3-5 \mathrm{~dB}$ relative to the bulk method. We do not have 


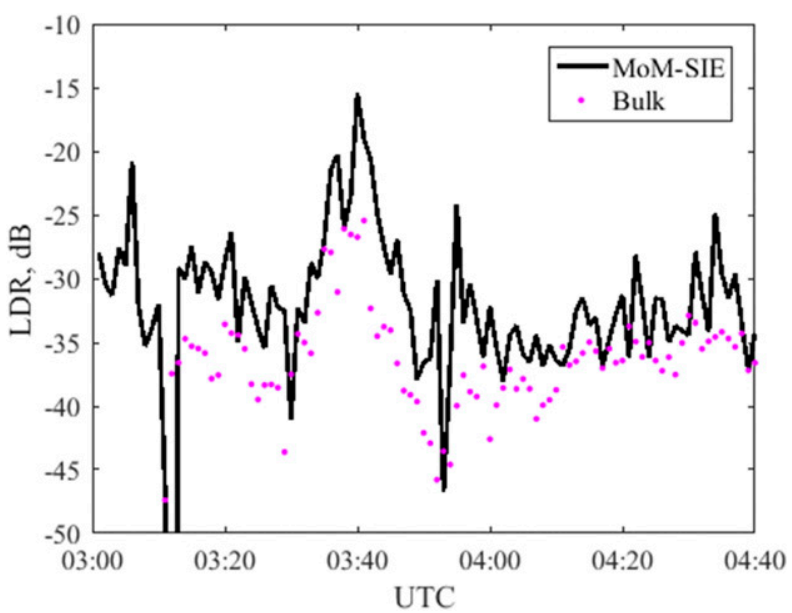

FIG. 7. LDR computation for incident $\phi=50^{\circ}$ and 1 -min averaging.

radar data to compare against, as the ARMOR radar is not configured for cross-polar reflectivity measurement. However, it is possible to use an analytical equation relating LDR, $Z_{\mathrm{dr}}, \rho_{\mathrm{hv}}, \delta$, and the standard deviation of the canting angle $\left(\sigma_{\beta}\right)$ (Jameson 1987) to illustrate the consistency between the dip in $\rho_{\mathrm{hv}}$ and the peak in LDR from MoM-SIE calculations during the line convection passage. Using (3.232) from Bringi and Chandrasekar (2001) and setting the values of $Z_{\mathrm{dr}}=3 \mathrm{~dB}, \rho_{\mathrm{hv}}=0.8$, $\delta=5^{\circ}$, and $\sigma_{\beta}=10^{\circ}$ predicts an LDR of $-19 \mathrm{~dB}$, which is consistent with the MoM-SIE peak LDR of $-17 \mathrm{~dB}$, coinciding with the dip in $\rho_{\mathrm{hv}}$ to 0.82 (close to the radarmeasured dip of 0.8). On the other hand, under the same conditions, setting LDR in (3.232) to the bulk peak value of $-26 \mathrm{~dB}$ predicts a much larger $\rho_{\mathrm{hv}}=0.97$ (the dip in bulk $\rho_{\mathrm{hv}}$ is only to 0.96 ). Thus, assuming that the radar-measured dip in $\rho_{\mathrm{hv}}$ to 0.8 is accurate, we can infer that the MoM-SIE-calculated LDR peak of $-17 \mathrm{~dB}$ is more consistent with the radar dip in $\rho_{\mathrm{hv}}$ than the bulk peak of $-25 \mathrm{~dB}$. It follows that the large MoM-SIE LDR values in the line convection are due to the enhanced variance in drop shapes resulting from the presence of asymmetric drops that cannot be modeled using the bulk method. Over the entire 100-min event, Table 1 shows the relative frequency of occurrence of MoM-SIE and bulk LDR values in 5-dB bins. The modal value (at the bin center) of LDR for the MoM-SIE method and the bulk method are, respectively, -32.5 and $-37.5 \mathrm{~dB}$, with the MoM-SIE showing positive skewness.

It should also be noted that because the scattering amplitudes for asymmetric drops exhibit $\phi$ dependence, as we saw earlier in Fig. 3, it is necessary to choose the correct $\phi$ angle, particularly for the $Z_{\mathrm{dr}}$ calculations. In our case the azimuth angle from the radar to the 2DVD site was $52^{\circ}$, and our reconstruction of drops is referenced to true north (since the 2DVD was aligned in such a way that this
TABLE 1. Relative frequency of occurrence (\%) of MoM-SIE and bulk LDR values $(\mathrm{dB})$ in 5-dB bins computed with drop-bydrop MoM-SIE and bulk T-matrix methods.

\begin{tabular}{ccc}
\hline \hline Range of LDR & T matrix & MoM-SIE \\
\hline$<-40$ & 21.3 & 3.96 \\
-40 to -35 & 49.2 & 19.8 \\
-35 to -30 & 19.7 & 46.54 \\
-30 to -25 & 9.8 & 19.8 \\
-25 to -20 & 0.0 & 7.92 \\
-20 to -15 & 0.0 & 1.98 \\
-15 to -10 & 0.0 & 0.0 \\
$>-10$ & 0.0 & 0.0 \\
\hline
\end{tabular}

criterion was met); hence, we chose the same value for $\phi$. In Fig. 8 we compare the single-particle $Z_{\mathrm{dr}}$ for $\phi=50^{\circ}$, $110^{\circ}$, and $180^{\circ}$. Also shown is the [1:1] line. As can be seen, the correlation is high with a negligible bias in both plots, and as a result any significant $\phi$ dependence would not be expected when the overall $Z_{\mathrm{dr}}$ is calculated for all drops over a 1-min integration period.

A limitation of the drop reconstruction procedure is that for a given $z=$ constant plane, there are only four points available from the two orthogonal cameras, and the fourellipse quarters constructed in this plane can have uncertainties in between these four points. However, because raindrops do not have sharp discontinuities (unlike snow particles), and because they are homogeneous, the resulting errors in the corresponding scattering calculations are not likely to be significant. Another limitation is that deskewing asymmetric drop shapes relies on the accuracy of estimating the horizontal drop speed and direction. In the future we will evaluate whether the wind speed and direction measured at the height of the 2DVD sensor area can be used to deskew asymmetric drops.

Another possible source of errors when comparing disdrometer-based estimates against radar measurements is the different spatial scales of the radar and "point" 2DVD measurements as well as the height of the radar pulse volume above the surface $(340 \mathrm{~m}$ in our case). At short ranges considered herein $(15 \mathrm{~km})$, the temporal decorrelation between radar and 2DVD is likely to be constrained, as evident in Fig. 6. It is well known that surface point measurements cannot be representative of the radar pixel, which is often quantified in terms of point-to-area variance (Ciach and Krajewski 1999; Thurai et al. 2012), which depends on the spatial correlation function of the observable used in the comparison. Other sources of errors include radar-measurement errors and disdrometer-sampling errors. However, it is beyond the scope of this paper to quantify the error variances arising from such error sources [we refer to Thurai et al. (2012) for variance analysis using ARMOR and 2DVD data]. 

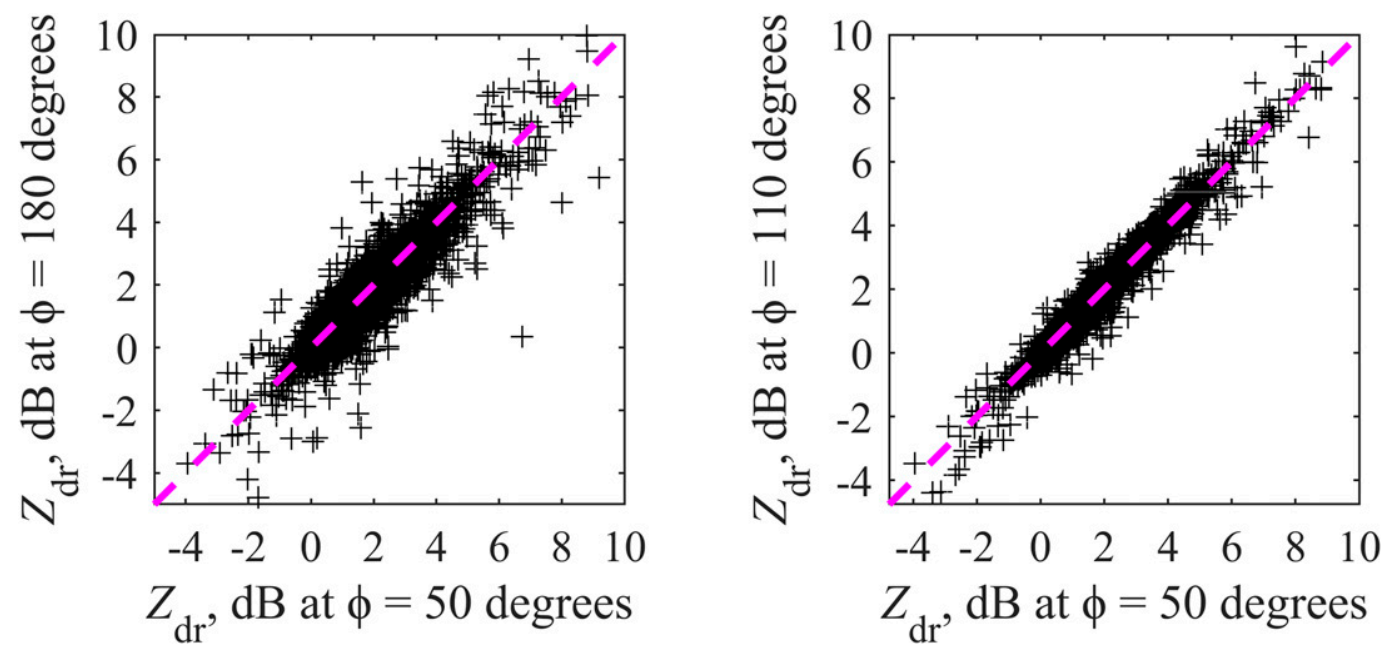

FIG. 8. Single-particle $Z_{\mathrm{dr}}$ comparison for particles with $D_{\mathrm{eq}} \geq 2 \mathrm{~mm}$ for (left) $\phi=50^{\circ} \mathrm{vs} \phi=180^{\circ}$ and (right) $\phi=50^{\circ}$ vs $\phi=110^{\circ}$. The [1:1] line is indicated (purple dashed line).

\section{Discussion and conclusions}

The bulk method of simulating radar observables such as $Z_{\mathrm{h}}, Z_{\mathrm{dr}}, K_{\mathrm{dp}}, \rho_{\mathrm{hv}}$, and LDR in rain involves simplifying assumptions, the main one being related to neglecting the effect of variance in shapes resulting from the presence of asymmetric drops, if in fact they occur in significant proportion to the more ubiquitous equilibrium (axisymmetric) shapes. There are very few computations of radar observables that explicitly account for variance in drop shapes. Keat et al. (2016) used the data from an artificial rain experiment reported in Thurai and Bringi (2005) to simulate steady-state axisymmetric drop oscillations (assuming a Gaussian axis ratio pdf) and its effects on $\rho_{\mathrm{hv}}$ and $Z_{\mathrm{dr}}$ using a gamma distribution of drop sizes (DSD) and Rayleigh-Gans theory. Their goal was to retrieve the shape parameter $\mu$ of the gamma DSD from radar measurements of $\left[\rho_{\mathrm{hv}} ; \mathrm{Z}_{\mathrm{dr}}\right]$. Their bulk simulations indicated drop oscillations had to be taken into account in order for the radar-based retrieval of $\mu$ to be unbiased. Thurai et al. (2009b) used 2DVD measurements to simulate drop-bydrop scattering but assumed symmetric shapes and canting angles derived from the deskewing procedure as in Huang et al. (2008). The agreement with ARMOR radar measurements was good, but they found significant differences in $\mathrm{Z}_{\mathrm{dr}}$ and $\rho_{\mathrm{hv}}$ when compared with bulk methods in one convective rain event.

To the best of our knowledge this study is the first polarimetric scattering analysis of a line convection rain event based on drop-by-drop scattering computations by means of a higher-order method of moments in a surface integral equation formulation, with asymmetric drop geometries being reconstructed from 2DVD measurements. We have compared MoM-SIE surface model discretization results for an example asymmetric drop with an equivolume drop diameter $D_{\text {eq }}=4.81 \mathrm{~mm}$ (in Fig. 1) at S, C, and $\mathrm{X}$ bands with volumetric discretization results by an industry standard finite element method-based code (HFSS), showing excellent agreement between the two methods. The single-particle $Z_{\mathrm{dr}}$ values showed variability during the passage of the line convection over the 2DVD site with a coefficient of variation (when $Z_{\mathrm{dr}}$ is expressed as a ratio) of around 0.5 (for drops $>3 \mathrm{~mm}$ ), which confirms that the variance of drop shapes resulting from asymmetric drops can be an important factor in this particular case. Note that before and after passage of the line convection, the shape variability was sharply reduced.

Drop-by-drop scattering calculations based on a 1-min integration of the covariance matrix elements were performed for the 100-min event passage over the 2DVD site using the MoM-SIE method and the bulk method. The simulated radar observables were compared with ARMOR radar data extracted from range gates surrounding the 2DVD location. The $Z_{\mathrm{h}}, Z_{\mathrm{dr}}$, and $K_{\mathrm{dp}}$ were found to be in good agreement between MoM-SIE, the bulk calculations, and the extracted ARMOR data during the line convection passage as well as before and after the passage. However, the bulk method could not simulate the significant lowering of $\rho_{\mathrm{hv}}$ during the line convection with a dip to 0.8 as measured by radar. The MoM-SIE calculations were able to simulate the dip to 0.8 , indicating that the lowered values were a result of variance in shapes resulting from asymmetric drops. The radar differential phase data showed no evidence of a backscatter differential phase (estimated $\left.\delta<3^{\circ}-5^{\circ}\right)$ within the line convection and neither did the single-drop MoM-SIE calculations $\left(\delta<5^{\circ}\right)$, so this effect could not have contributed to the lowering of $\rho_{\mathrm{hv}}$. 
We also computed the LDR using the drop-by-drop MoM-SIE and the bulk methods. During the line convection passage over the 2DVD, the MoM-SIE LDR values peaked at $-17 \mathrm{~dB}$, whereas the bulk LDR was around $8 \mathrm{~dB}$ lower $(-25 \mathrm{~dB})$. Examination of an analytic expression relating the polarimetric variables showed that the MoM-SIE LDR peak of $-17 \mathrm{~dB}$ was consistent with the dip in $\rho_{\mathrm{hv}}$ to 0.8 (the latter in agreement with the radar-observed dip). However, the bulk LDR of $-25 \mathrm{~dB}$ was not consistent with the observed $\rho_{\text {hv }}$ dip, with the analytic expression giving a much higher $\rho_{\mathrm{hv}}$ value of 0.97 consistent with the calculated bulk value of 0.96 . Since the ARMOR radar was not configured for LDR measurements, we could not compare them with the simulated values. Over the full 100-min event, the modal MoM-SIE LDR values were around $-32.5 \mathrm{~dB}$, whereas they were around $-37.5 \mathrm{~dB}$ for the bulk method. Radars with a modest dual-polarized antenna with a system LDR limit of $-25 \mathrm{~dB}$ (e.g., phased-array airborne radars) could easily detect the LDR peak of $-17 \mathrm{~dB}$. However, to detect an LDR of $-32.5 \mathrm{~dB}$, a welldesigned antenna capable of a system LDR limit of $-36 \mathrm{~dB}$ would be required (the U.K. C-band operational radars approach the -36 - $\mathrm{dB}$ system limit and they routinely measure LDR to detect wet snow aloft; Sandford et al. 2017).

As has been mentioned in earlier publications (Thurai et al. 2013, 2014), 2DVD data examined during most of the rain events showed that the drop shapes conform to the "most probable" shapes arising from the steadystate axisymmetric oscillation mode, which can be regarded as the background state. Asymmetric shapes occur when the background state is perturbed because of transverse or horizontal modes mixed in, which is termed mixed-mode oscillations (Beard et al. 2010). The line convection system considered here is one of the few exceptions where a significant proportion $(\approx 30 \%)$ of asymmetric drops was detected only within the line convection but not outside it. Currently, there is no theoretical framework to identify the conditions under which mixed-mode oscillations may occur in a persistent manner. For now we have to rely on 2DVD data to first detect the presence of a significant proportion of asymmetric drops in the rain shaft and subsequently to evaluate the conditions under which deviations from the most probable axisymmetric drop shapes occur. Based on this study, the most impact would be on the quantitative use of $\rho_{\mathrm{hv}}$ and LDR, with much less impact on $Z_{\mathrm{dr}}$, and a negligible impact on $Z_{\mathrm{h}}$ and $K_{\mathrm{dp}}$.

Acknowledgments. This work was supported by the National Science Foundation under Grant AGS-1431127. We also acknowledge the University of Alabama in
Huntsville for providing the ARMOR radar observations used in this NSF study.

The 2DVD data (in the form hyd/hd files) used in this study can be made available upon request from Dr. M. Thurai (merhala@colostate.edu) or Dr. P.N. Gatlin (patrick.gatlin@nasa.gov) or Dr. M. Schönhuber (michael. schoenhuber@joanneum.at). ARMOR radar data are archived at the University of Alabama in Huntsville and can be made available.

\section{REFERENCES}

ANSYS, 2017: High Frequency Structural Simulator (HFSS), finite element method (FEM). ANSYS, http://www.ansys.com/ products/electronics/ansys-hfss.

Barber, P., and C. Yeh, 1975: Scattering of electromagnetic waves by arbitrarily shaped dielectric bodies. Appl. Opt., 14, 28642872, https://doi.org/10.1364/AO.14.002864.

Beard, K. V., and C. Chuang, 1987: A new model for the equilibrium shape of raindrops. J. Atmos. Sci., 44, 1509-1524, https:// doi.org/10.1175/1520-0469(1987)044<1509:ANMFTE >2.0.CO;2.

_, V. N. Bringi, and M. Thurai, 2010: A new understanding of raindrop shape. Atmos. Res., 97, 396-415, https://doi.org/ 10.1016/j.atmosres.2010.02.001.

Brandes, E. A., G. Zhang, and J. Vivekanandan, 2004: Drop size distribution retrieval with polarimetric radar: Model and application. J. Appl. Meteor., 43, 461-475, https://doi.org/ 10.1175/1520-0450(2004)043<0461:DSDRWP>2.0.CO;2.

Bringi, V. N., and V. Chandrasekar, 2001: Polarimetric Doppler Weather Radar: Principles and Applications. Cambridge University Press, 636 pp.

—, M. Thurai, K. Nakagawa, G. J. Huang, T. Kobayashi, A. Adachi, H. Hanado, and S. Sekizawa, 2006: Rainfall estimation from C-band polarimetric radar in Okinawa, Japan: Comparisons with $2 \mathrm{D}$-video disdrometer and $400 \mathrm{MHz}$ wind profiler. J. Meteor. Soc. Japan, 84, 705-724, https://doi.org/10.2151/jmsj.84.705.

Carey, L. D., and W. A. Petersen, 2015: Sensitivity of C-band polarimetric radar-based drop size estimates to maximum diameter. J. Appl. Meteor. Climatol., 54, 1352-1371, https://doi.org/10.1175/ JAMC-D-14-0079.1.

Chobanyan, E., N. J. Šekeljić, A. B. Manić, M. M. Ilić, V. N. Bringi, and B. M. Notaroš, 2015: Efficient and accurate computational electromagnetics approach to precipitation particle scattering analysis based on higher-order method of moments integral equation modeling. J. Atmos. Oceanic Technol., 32, 17451758, https://doi.org/10.1175/JTECH-D-15-0037.1.

Ciach, G. J., and W. F. Krajewski, 1999: On the estimation of radar rainfall error variance. Adv. Water Resour., 22, 585-595, https://doi.org/10.1016/S0309-1708(98)00043-8.

Crowe, C. C., C. J. Schultz, M. R. Kumjian, L. D. Carey, and W. A. Petersen, 2012: Use of dual-polarization signatures in diagnosing tornadic potential. Electron. J. Oper. Meteor., 13, 57-78.

Djordjević, M., and B. M. Notaroš, 2004: Double higher order method of moments for surface integral equation modeling of metallic and dielectric antennas and scatterers. IEEE Trans. Antennas Propag., 52, 2118-2129, https://doi.org/10.1109/ TAP.2004.833175.

Huang, G., V. N. Bringi, and M. Thurai, 2008: Orientation angle distributions of drops after an $80-\mathrm{m}$ fall using a 2D video disdrometer. J. Atmos. Oceanic Technol., 25, 1717-1723, https:// doi.org/10.1175/2008JTECHA1075.1. 
Hubbert, J., and V. N. Bringi, 1995: An iterative filtering technique for the analysis of copolar differential phase and dual-frequency radar measurements. J. Atmos. Oceanic Technol., 12, 643-648, https:// doi.org/10.1175/1520-0426(1995)012<0643:AIFTFT>2.0.CO;2.

Jameson, A. R., 1983: Microphysical interpretation of multiparameter radar measurements in rain. Part I: Interpretation of polarization measurements and estimation of raindrop shapes. J. Atmos. Sci., 40, 1792-1802, https://doi.org/10.1175/ 1520-0469(1983)040<1792:MIOMPR > 2.0.CO;2.

— 1985: Microphysical interpretation of multiparameter radar measurements in rain. Part III: Interpretation and measurement of propagation differential phase shift between orthogonal linear polarizations. J. Atmos. Sci., 42, 607-614, https:// doi.org/10.1175/1520-0469(1985)042<0607:MIOMRM>2.0.CO;2.

_ 1987: Relations among linear and circular polarization parameters measured in canted hydrometeors. J. Atmos. Oceanic Technol., 4, 634-646, https://doi.org/10.1175/1520-0426(1987)004<0634: RALACP $>2.0 . C O ; 2$.

Keat, W. J., C. D. Westbrook, and A. J. Illingworth, 2016: Highprecision measurements of the copolar correlation coefficient: Non-Gaussian errors and retrieval of the dispersion parameter $\mu$ in rainfall. J. Appl. Meteor. Climatol., 55, 1615-1632, https:// doi.org/10.1175/JAMC-D-15-0272.1.

Klopf, E. M., N. J. Sekeljić, M. M. Ilić, and B. M. Notaroš, 2012: Optimal modeling parameters for higher order MoM-SIE and FEM-MoM electromagnetic simulations. IEEE Trans. Antennas Propag., 60, 2790-2801, https://doi.org/10.1109/ TAP.2012.2194669.

Lee, G. W., and I. Zawadzki, 2005: Variability of drop size distributions: Noise and noise filtering in disdrometric data. J. Appl. Meteor., 44, 634-652, https://doi.org/10.1175/JAM2222.1.

Meischner, P. F., V. N. Bringi, D. Heimann, and H. Höller, 1991: A squall line in southern Germany: Kinematics and precipitation formation as deduced by advanced polarimetric and Doppler radar measurements. Mon. Wea. Rev., 119, 678-701, https:// doi.org/10.1175/1520-0493(1991)119<0678:ASLISG >2.0.CO;2.

Mishchenko, M. I., L. D. Travis, and D. W. Mackowski, 1996: T-matrix computations of light scattering by nonspherical particles: A review. J. Quant. Spectrosc. Radiat. Transfer, 55, 535-575, https://doi.org/ 10.1016/0022-4073(96)00002-7.

Notaroš, B. M., 2008: Higher order frequency-domain computational electromagnetics. IEEE Trans. Antennas Propag., 56, 2251-2276, https://doi.org/10.1109/TAP.2008.926784.

Petersen, W. A., K. R. Knupp, D. J. Cecil, and J. R. Mecikalski, 2007: The University of Alabama Huntsville THOR Center instrumentation: Research and operational collaboration. 33rd Int. Conf. on Radar Meteorology, Cairns, QLD, Australia, Amer. Meteor. Soc., 5.1, https://ams.confex.com/ams/33Radar/ webprogram/Paper123410.html.

Sandford, C., A. Illingworth, and R. Thompson, 2017: The potential use of the linear depolarization ratio to distinguish between convective and stratiform rainfall to improve radar rain-rate estimates. J. Appl. Meteor. Climatol., 56, 29272940, https://doi.org/10.1175/JAMC-D-17-0014.1.

Schönhuber, M., W. L. Randeu, H. E. Urban, and J. P. V. Poiares Baptista, 2000: Field measurements of raindrop orientation angles. Proc. AP2000 Millennium Conf. on Antennas and Propagation, Davos, Switzerland, IEE, 4 pp.

—, G. Lammer, and W. L. Randeu, 2008: The 2D-video-disdrometer. Precipitation: Advances in Measurement, Estimation and Prediction, S. C. Michaelides, Ed., Springer, 3-31.

, M. Schwinzerl, and G. Lammer, 2016: 3D reconstruction of 2DVD-measured raindrops for precise prediction of propagation parameters. 2016 10th European Conference on Antennas and Propagation (EuCAP), IEEE, 3403-3406, https://doi.org/ 10.1109/EuCAP.2016.7481929.

Schuur, T. J., A. V. Ryzhkov, D. S. Zrnić, and M. Schönhuber, 2001: Drop size distributions measured by a 2-D video disdrometer: Comparison with dual-polarization radar data. J. Appl. Meteor., 40, 1019-1034, https://doi.org/10.1175/1520-0450(2001)040<1019: DSDMBA $>2.0 . \mathrm{CO} ; 2$.

Schwinzerl, M., M. Schönhuber, G. Lammer, and M. Thurai, 2016: $3 \mathrm{D}$ reconstruction of individual raindrops from precise ground-based precipitation measurements. EMS Annual Meeting Abstracts, Vol. 13, Abstract EMS2016-601, http:// meetingorganizer.copernicus.org/EMS2016/EMS2016-601.pdf.

Seliga, T. A., and V. N. Bringi, 1976: Potential use of radar differential reflectivity measurements at orthogonal polarizations for measuring precipitation. J. Appl. Meteor., 15, 69-76, https://doi.org/ 10.1175/1520-0450(1976)015<0069:PUORDR > 2.0.CO;2.

— , and - 1978: Differential reflectivity and differential phase shift: Applications in radar meteorology. Radio Sci., 13, 271275, https://doi.org/10.1029/RS013i002p00271.

Szakáll, M., K. Diehl, S. K. Mitra, and S. Borrmann, 2009: A wind tunnel study on the shape, oscillation, and internal circulation of large raindrops with sizes between 2.5 and $7.5 \mathrm{~mm}$. J. Atmos. Sci., 66, 755-765, https://doi.org/10.1175/2008JAS2777.1.

Thurai, M., and V. N. Bringi, 2005: Drop axis ratios from a 2D video disdrometer. J. Atmos. Oceanic Technol., 22, 966-978, https://doi.org/10.1175/JTECH1767.1.

- G. J. Huang, V. N. Bringi, W. L. Randeu, and M. Schönhuber, 2007: Drop shapes, model comparisons, and calculations of polarimetric radar parameters in rain. J. Atmos. Oceanic Technol., 24, 1019-1032, https://doi.org/10.1175/JTECH2051.1.

— , V. N. Bringi, M. Szakáll, S. K. Mitra, K. V. Beard, and S. Borrmann, 2009a: Drop shapes and axis ratio distributions: Comparison between 2D video disdrometer and wind-tunnel measurements. J. Atmos. Oceanic Technol., 26, 1427-1432, https://doi.org/10.1175/2009JTECHA1244.1.

$\longrightarrow,-$, and W. A. Petersen, 2009b: Rain microstructure retrievals using 2-D video disdrometer and C-band polarimetric radar. Adv. Geosci., 20, 13-18, https://doi.org/10.5194/ adgeo-20-13-2009.

,,-- L. D. Carey, P. Gatlin, E. Schultz, and W. A. Petersen, 2012: Estimating the accuracy of polarimetric radar-based retrievals of drop size distribution parameters and rain rate: An application of error variance separation using radarderived spatial correlations. J. Hydrometeor., 13, 1066-1079, https://doi.org/10.1175/JHM-D-11-070.1.

, W. A. Petersen, and P. N. Gatlin, 2013: Drop shapes and fall speeds in rain: Two contrasting examples. J. Appl. Meteor. Climatol., 52, 2567-2581, https://doi.org/10.1175/ JAMC-D-12-085.1.

,-- A. B. Manić, N. J. Sekeljić, and B. M. Notaroš, 2014: Investigating rain drop shapes, oscillation modes, and implications for radiowave propagation. Radio Sci., 49, 921-932, https://doi.org/10.1002/2014RS005503.

_ - S. Manić, M. Schönhuber, V. N. Bringi, and B. M. Notaroš, 2017: Scattering calculations at C-band for asymmetric raindrops reconstructed from 2D video disdrometer measurements. J. Atmos. Oceanic Technol., 34, 765-776, https://doi.org/ 10.1175/JTECH-D-16-0141.1.

Waterman, P. C., 1965: Matrix formulation of electromagnetic scattering. Proc. IEEE, 53, 805-812, https://doi.org/10.1109/ PROC.1965.4058. 